\title{
Nuovi approcci per lo studio delle migliori tecnologie disponibili a supporto dell' autorizzazione integrata ambientale per impianti di produzione di biometano
}

\author{
Noemi Cennamo ${ }^{1}$ \\ ${ }^{1}$ Affiliation not available
}

\begin{abstract}
L'Autorizzazione Integrata Ambientale (AIA) è il provvedimento che autorizza l'esercizio di un impianto o parte di esso, in cui si svolgono attività che potrebbero causare significativi impatti ambientali. Con l'AIA le imprese possono uniformarsi ai principi di IPPC che sta per Integrated Pollution Prevention and Control, individuando soluzioni tecniche, impiantistiche ed economiche, per una corretta gestione delle possibili forme di inquinamento. Per raggiungere elevati livelli di protezione dell'ambiente bisogna adottare le Migliori Tecniche Disponibili, in inglese Best Available Technology (BAT). Ad oggi in Italia non è ancora presente un metodo di studio delle migliori tecnologie disponibili, ma una diversa situazione si ha nel Regno Unito dove viene il software H1. Lo scopo del presente lavoro è individuare una metodologia di studio delle BAT per un impianto di trattamento della Frazione Organica dei Rifiuti Solidi Urbani (FORSU) e scarti ligneo-cellulosici per la produzione di biometano situato in Italia, applicando per lo stesso il software inglese.
\end{abstract}

\section{L'AIA nella legislazione italiana e inglese}

La Direttiva 96/61/CE (Direttiva IPPC) è stata recepita In Italia prima con il D. Lgs. 372/1999 “Attuazione della direttiva 96/61/CE relativa alla prevenzione e riduzione integrate dell'inquinamento" 
e poi con il D. Lgs. 59/2005 “Attuazione integrale della Direttiva 96/61/CE relativa alla prevenzione e riduzione integrate dell'inquinamento" ${ }^{1}$. Attualmente la norma di riferimento è il D. Lgs. 46/2014 che ha apportato modifiche al D. Lgs. 152/2006 (TUA) ${ }^{2}$. L'autorità competente del provvedimento di AIA è la Regione (secondo le disposizioni delle leggi regionali e provinciali) per gli impianti dell'allegato VIII alla Parte Seconda del D. Lgs. 152/06 e ss.mm.ii., mentre lo è il Ministero dell'Ambiente e della Tutela del Territorio e del Mare per quelli dell'allegato XII. L'AIA ha una durata di cinque anni (10 per gli impianti riportati al punto 6.6 dell'allegato VIII alla Parte Seconda), ma può essere estesa a sei o otto anni nel caso in cui l'impianto sia in possesso di certificazioni ambientali ISO 14001 e/o EMAS (Gruppo Maurizi). Sostituendo tutte le autorizzazioni in campo ambientale, l'AIA costituisce una semplificazione amministrativa. Per il suo rilascio, il gestore dell'impianto richiedente presenta una domanda firmata e con marca da bollo all'ufficio competente e una sintesi non tecnica con in allegato l'attestazione del pagamento delle spese di istruttoria e controllo di cui al D.M. 24.4.2008. L'autorità competente, entro 30 giorni dal ricevimento della domanda, comunica al gestore la data di avvio del procedimento il quale nei successivi 15 giorni deve pubblicare la notizia sul sito web. Nei 15 giorni dopo, l'autorità competente raccoglie le eventuali osservazioni e la Conferenza dei Servizi entro 150 giorni deve procedere con il rilascio o meno dell'autorizzazione. Essa però può richiedere integrazioni o specificazioni da presentare in un arco temporale massimo di 90 giorni. Un altro elaborato è la Relazione tecnica, un documento riassuntivo delle informazioni riportate nelle schede redatte dal tecnico abilitato che si compone di una parte descrittiva e di elaborati grafici. Per quanto riguarda il sistema di autorizzazioni ambientali del Regno Unito, questo ha subìto cambiamenti nel tempo. Dal 1995 il Paese si è dotato di un'unica autorità per le problematiche ambientali, costituendo un notevole vantaggio per il passaggio ad un approccio integrato, e nel 1999 il PPC "Pollution Prevention and Control Act" implementa la Direttiva IPPC. Ad oggi in letteratura sono scarse le applicazioni della Direttiva IPPC, per cui si fa riferimento all'iter tecnico-amministrativo della precedente direttiva (IPC) che ha posto le basi per il nuovo sistema autorizzativo (ISPRA). Anche in 
questo caso il gestore dell'impianto presenta la domanda fornendo dati quali nome, indirizzo per la corrispondenza, dati relativi alla registrazione dell'impianto, la sede in cui sarà svolto il processo, descrizione del processo, se l'impianto è nuovo o esistente e le sue caratteristiche, cosa si produce e in che quantità, ecc. Questa viene pubblicata su una rivista locale, di cui una copia depositata nel registro pubblico tenuto dall'Her Majesty's Industrial Pollution Inspectorate (HMIPI) ovvero Ispettorato per l'inquinamento industriale, e altre copie vengono inviate ad enti e autorità locali che insieme al pubblico possono produrre le osservazioni in massimo 28 giorni. Se non ve ne sono l'autorizzazione viene rilasciata, ma sarà comunque soggetta a revisione dopo quattro anni, momento in cui l'ispettore terrà conto dei risultati dei monitoraggi, dei cambiamenti economici e tecnologici, al fine di promuovere l'adozione di tecnologie pulite 345678

\section{Comparto polifunzionale di trattamento dei rifiuti per la produ-}

\section{zione di biometano: il confronto puntuale con le BAT}

L'impianto oggetto di studio è situato nell'Italia settentrionale. Mediante un trattamento di tipo anaerobico-aerobico di FORSU e scarti di verde e potature ${ }^{9}$, produce compost di qualità e biometano a partire dal biogas del trattamento anaerobico ${ }^{10}{ }^{11}$. Particolarmente importante risulta la produzione di biometano che consente di evitare il consumo di oltre 6000 tonnellate di petrolio all'anno come combustibile fossile, pari a circa 14,000 tonnellate di anidride carbonica evitate. Esso viene immesso nella rete di distribuzione gas per poi essere utilizzato per alimentare mezzi pubblici operanti sul territorio, veicoli privati, ecc. e consente di rispettare il concetto di sviluppo sostenibile ${ }^{12} 13$ (Dal rifiuto organico al biometano - Progetto biometano HERAmbiente). Le emissioni odorigene rappresentano l'aspetto critico, continuamente denunciate dagli abitanti delle

zone limitrofe 14151617181920212223 . Esse sono state monitorate con il modello di dispersione Calpuff ${ }^{24}$. 
L'AIA per l'impianto in esame presenta tre allegati (Portale AIA Emilia-Romagna):

1. Allegato I: "Condizioni dell'Autorizzazione Integrata Ambientale per il Comparto polifunzionale di trattamento rifiuti";

2. Allegato II: "Tabelle BAT";

3. Allegato III: "Parere urbanistico-edilizio del Comune".

Di grande interesse risulta il secondo allegato nel quale, in forma tabellare, viene valutata l'applicazione o meno delle BAT da parte dell'impianto. Le BAT considerate sono definite nell'ambito dei Bref "BAT reference documents" per gli impianti di trattamento dei rifiuti, periodicamente aggiornati e pubblicati sul sito della Commissione Europea (Brefs - European Commission site). Dallo studio dell'allegato è emerso che sono state applicate quasi la totalità delle BAT riferite a questa tipologia di impianto, cosa che giustifica dunque il rilascio dell'AIA ${ }^{25}$.

Il software H1 diffuso nel Regno Unito costituisce uno strumento semplificativo messo a disposizione del tecnico che deve eseguire o una valutazione di impatto ambientale di un impianto nel suo complesso, o deve effettuare un'analisi delle diverse opzioni che costituiscono i sistemi di abbattimento delle emissioni (scarichi) di un impianto, al fine di determinare le BAT (Software H1, Department of Agricolture, Environment and Rural Affairs site).

Poiché lo scopo del presente lavoro è individuare una nuova metodologia di studio delle BAT, il software è stato impiegato per il perseguimento del secondo obiettivo, per un impianto di produzione di biometano italiano. Il software prevede sei step, ma per lo scopo sono stati completati solo i primi quattro:

1. Step 1: Descrivere lo scopo e le opzioni

2. Step 2: Inventario delle emissioni 
3. Step 3: Quantificare gli impatti

4. Step 4: Comparare gli impatti tra le opzioni

I sistemi di abbattimento delle emissioni dell'impianto oggetto di studio riguardano soltanto le emissioni in atmosfera, per cui la prima opzione inserita, denominata "base-case", è la tecnica che effettivamente viene implementata dall'impianto ed è il biofiltro. Le opzioni successive rappresentano delle alternative alla prima, e sono state individuate facendo riferimento ai Bref. Per ogni opzione il software richiede informazioni come ad esempio punti di emissione, portata emessa, velocità di efflusso, rifiuti prodotti, sostanze rilasciate e così via, che vengono elaborate e presentate in istogrammi per comparare parametri quali la produzione di rifiuti o di $\mathrm{CO}_{2}$, gli effetti del rilascio di sostanze nell'aria a breve e a lungo termine, delle diverse opzioni considerate, rendendo oggettiva l'identificazione della migliore opzione. Dalla comparazione però potrebbero non emergere sostanziali differenze tra le opzioni e allora potrebbe non essere immediato individuare la migliore. In questo caso si procede con il completamento di altri due step successivi, dedicati ad un'analisi dei costi associati alle opzioni analizzate. Sono diffusi diversi sistemi di supporto di analisi delle alternative ad esempio, anche per le centrali elettriche sostenibili ${ }^{26}$.

\section{Conclusioni}

Il presente lavoro ha riguardato l'utilizzo di un software impiegato nel Regno Unito, denominato H1 Software Tool, per lo studio delle BAT di un impianto di produzione di biometano situato nell'Italia settentrionale. L'obiettivo è definire una metodologia di studio delle BAT, attualmente assente in Italia. Nello studio è stato descritto prima il procedimento di AIA nella legislazione italiana e inglese, poi l'impianto oggetto di studio, e nello specifico il secondo allegato all'AIA: "Tabelle BAT". Tale allegato segnala l'applicazione o meno delle migliori tecnologie disponibili (definite dai Bref per gli impianti di trattamento dei rifiuti) da parte dell'impianto, con una descri- 
zione delle attività condotte ad esse riferite. La procedura di valutazione delle BAT del software inglese invece, non si limita solo a valutarne l'applicazione o meno, ma considerando insieme le tecniche che un impianto potrebbe impiegare in maniera alternativa, consente di scegliere la migliore grazie ad un confronto oggettivo, poiché basato su parametri numerici come emissioni (in termini di portata), produzione di rifiuti, produzione di $\mathrm{CO}_{2}$, effetti a lungo e a breve termine delle emissioni. In virtù dell'oggettività che caratterizza la procedura inglese di valutazione delle BAT, sarebbe da preferirsi quest'ultima rispetto a quella italiana, che può essere concepita anche come strumento di giustificazione/validazione delle scelte tecnologiche adottate dell'impianto.

\section{References}

1.Benedusi, L. L'autorizzazione integrata ambientale. in Gestione ambientale (TuttoAmbiente, 2019).

2.Bertuzzi, R. \& Carbone, N. Le modifiche all'AIA introdotte dal D.Lvo 46/2014. TuttoAmbiente.

3.Naddeo, V. \& Korshin, G. Water energy and waste: The great European deal for the environment. Science of The Total Environment 764, 142911 (2021).

4.Zarra, T., Galang, M. G., Ballesteros, F., Belgiorno, V. \& Naddeo, V. Environmental odour management by artificial neural network - A review. Environment International 133, 105189 (2019).

5.Hasan, S. W., Liu, H., Naddeo, V., Puig, S. \& Yip, N. Y. Environmental technologies for the sustainable development of the water and energy sectors - IWA Publishing. (https: / /www.iwapublishing.com/books/9781789062311/environmentaltechnologies-sustainable-development-water-and-energy-sectors, 2020). 
6.Borea, L., Zarra, T., Zablocka, J. B., Belgiorno, V. \& Naddeo, V. Water-Energy Nexus: Evaluation of the Environmental Impact on the National and International Scenarios. ADVANCES IN SCIENCE, TECHNOLOGY \& INNOVATION (2020).

7.Naddeo, V., Balakrishnan, M. \& Choo, K. Frontiers in Water-Energy-Nexus-Nature-Based Solutions, Advanced Technologies and Best Practices for Environmental Sustainability. (2020).

8.Cartolano, V., Senatore, V., Naddeo, V. \& Anderson, M. A. Performance analysis of a capacitive deionization stack for brackish water desalination. Desalination (2021).

9.Naddeo, V. \& Taherzadeh, M. J. Biomass valorization and bioenergy in the blue circular economy. Biomass and Bioenergy 149, 106069 (2021).

10.Cesaro, A., Naddeo, V., Amodio, V. \& Belgiorno, V. Enhanced biogas production from anaerobic codigestion of solid waste by sonolysis. Ultrasonics Sonochemistry 19, 596-600 (2012).

11.Naddeo, V., Cesaro, A., Amodio, V. \& Belgiorno, V. ANAEROBIC CO-DIGESTION OF MUNICIPAL SOLID WASTE WITH ULTRASOUND PRE-TREATMENT. in (2009).

12.Senatore, V. et al.. Innovative membrane photobioreactor for sustainable $\mathrm{CO} 2$ capture and utilization. Chemosphere 273, 129682 (2021).

13.Nesticò, A., Elia, C. \& Naddeo, V. Sustainability of urban regeneration projects: Novel selection model based on analytic network process and zero-one goal programming. Land Use Policy 99, 104831 (2020).

14.Zarra, T., Naddeo, V. \& Belgiorno, V. Characterization of odours emitted by liquid waste treatment plants (LWTPs). Issue 4 18, 721-727 (2016).

15.Naddeo, V., Zarra, T., Oliva, G. \& Cardona, G. Odour impact assessment of a large municipal solid waste landfill under different working phases. Issue 3 20, 654-658 (2018).

16.Zarra, T., Naddeo, V. \& Belgiorno, V. A novel tool for estimating the odour emissions of com- 
posting plants in air pollution management. Global Nest 11, (2009).

17.Zarra, T., Reiser, M., Naddeo, V., Belgiorno, V. \& Kranert, M. A Comparative and Critical Evaluation of Different Sampling Materials in the Measurement of Odour Concentration by Dynamic Olfactometry —Chemical Engineering Transactions. Italian Association of Chemical Engineering 30, (2012).

18.Naddeo, V., Zarra, T., Giuliano, S. \& Belgiorno, V. Odour Impact Assessment in Industrial Areas. Chemical Engineering Transaction 30, (2012).

19.Viccione, G., Zarra, T., Giuliani, S., Naddeo, V. \& Belgiorno, V. Performance Study of E-Nose Measurement Chamber for Environmental Odour Monitoring. Italian Association of Chemical Engineering 30, (2012).

20.Zarra, T., Giuliani, S., Naddeo, V. \& Belgiorno, V. Control of odour emission in wastewater treatment plants by direct and undirected measurement of odour emission capacity. Water Science and Technology 66, 1627-1633 (2012).

21.Zarra, T., Naddeo, V., Giuliani, S. \& Belgiorno, V. Optimization of Field Inspection Method for Odour Impact Assessment — Chemical Engineering Transactions. Italian Association of Chemical Engineering 23, (2011).

22.Oliva, G. et al. Next-generation of instrumental odour monitoring system (IOMS) for the gaseous emissions control in complex industrial plants. Chemosphere 271, 129768 (2021).

23.Zarra, T., Galang, M. G. K., Ballesteros, F. C., Belgiorno, V. \& Naddeo, V. Instrumental Odour Monitoring System Classification Performance Optimization by Analysis of Different PatternRecognition and Feature Extraction Techniques. Sensors 21, 114 (2020).

24.Naddeo, V., Zarra, T., Oliva, G., Chiavola, A. \& Vivarelli, A. Environmental Odour Impact Assessment of Landfill Expansion Scenarios: Case Study of Borgo Montello (Italy) - Chemical 
Engineering Transactions.

25.Naddeo, V., Belgiorno, V., Zarra, T. \& Scannapieco, D. Dynamic and embedded evaluation procedure for strategic environmental assessment. Land Use Policy 31, 605-612 (2013).

26.Scannapieco, D., Naddeo, V. \& Belgiorno, V. Sustainable power plants: A support tool for the analysis of alternatives. Land Use Policy 36, 478-484 (2014). 


\section{Figure Captions}

Figure 1. Biocarburante (fonte: Biocarburante da scarti organici: Hera vuole adottare una tecnologia Eni).

Figure 2. Copertina del Software H1 


\section{Figures}

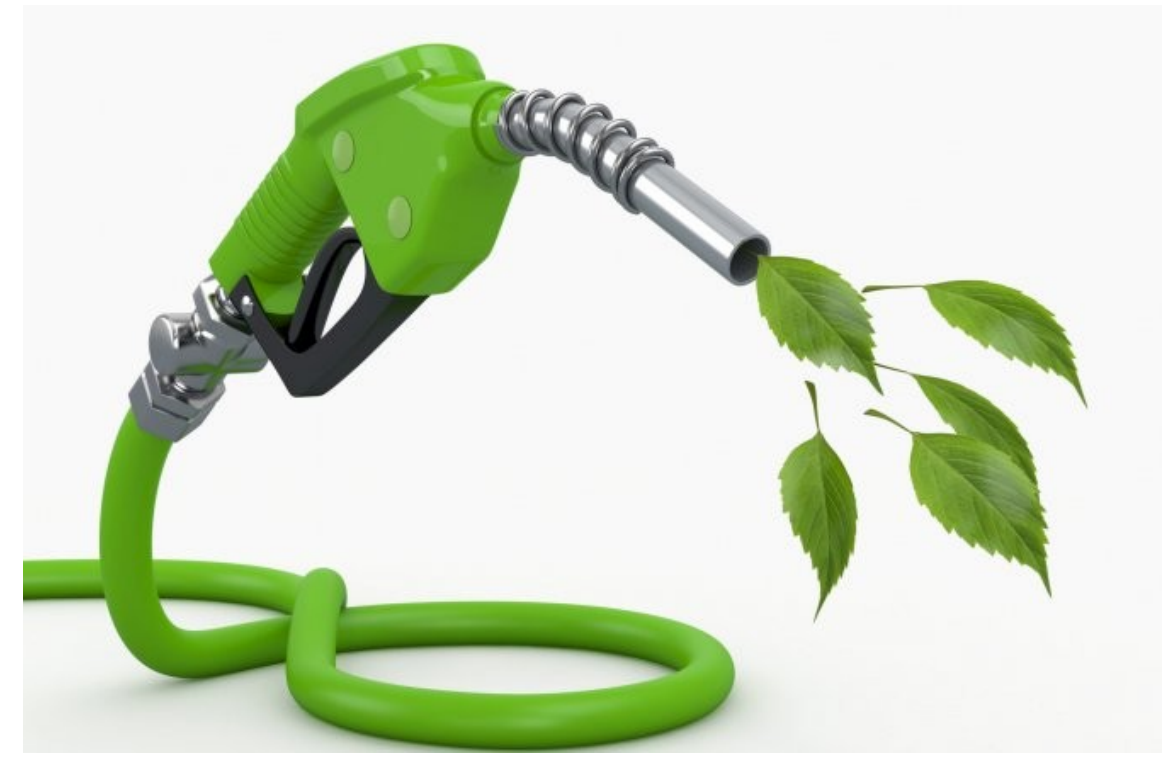

Figure 1: Biocarburante (fonte: Biocarburante da scarti organici: Hera vuole adottare una tecnologia Eni).

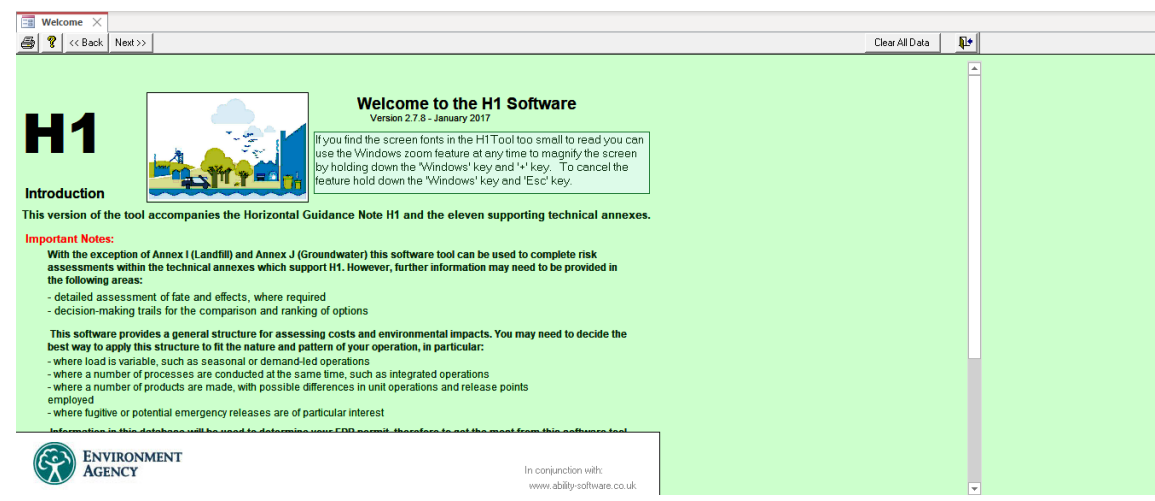

Figure 2: Copertina del Software H1 